\title{
Cascade of period doubling bifurcations and large stochasticity in the motions of a compass $(*)$
}

\author{
V. Croquette and C. Poitou \\ Service de Physique du Solide et de Résonance Magnétique, CEN Saclay, 91191 Gif sur Yvette Cedex, France
}

(Reçu le 3 juin 1981, accepté le 27 octobre 1981)

\begin{abstract}
Résumé. - Nous proposons un système mécanique très simple, constitué d'une boussole dans un champ alternatif périodique, qui permet l'observation de la stochasticité à grande échelle des systèmes hamiltoniens. Nous montrons que ce système présente aussi une cascade de dédoublements de période. Nous déterminons expérimentalement les seuils des premiers dédoublements.
\end{abstract}

\begin{abstract}
We propose a very simple mechanical device, consisting of a compass placed in a periodic oscillating field, which renders the observation of large scale stochasticity in Hamiltonian systems possible. We show that this device also exhibits a cascade of period doubling bifurcations. We determine experimentally the threshold of the first doublings.
\end{abstract}

If we place a compass, initially neglecting friction, in an oscillating magnetic field, perpendicular to the compass axis, we have a simple mechanical device, which models a well-known system : the synchronous bipolar motor. We might think that the possible motions of this compass are naturally a clockwise or a counterclockwise rotation. In fact, by doing so, we have not considered the non-linear property of this device, and if these two kinds of motion really exist, a multitude of different motions are also possible, some periodic, others chaotic.

For this device, the angle of rotation $\theta$ is governed by the equation :

$$
\ddot{\theta}=-F[\sin (\theta-\omega t)+\sin (\theta+\omega t)]
$$

with $F=\left(M . B_{0} / 2 J\right)$ and where $M$ and $J$ are respectively the magnetic and inertial momentum of the compass. $B_{0}$ is the magnitude of the magnetic field, oscillating at the frequency $(\omega / 2 \pi)$. A detailed study of this kind of equation $\left({ }^{1}\right)$ has been performed by D. Escande and F. Doveil [1] and also in [6,7].

Let us first consider the compass placed in a single rotating field. In the rotating frame, equation (1) becomes :

$$
\ddot{\theta}_{1}=-F\left[\sin \theta_{1}\right]
$$

(*) La version française de cet article a été proposée pour publication aux Comptes Rendus de l'Académie des Sciences.

( $\left.{ }^{1}\right)$ Equation (1) also models a phase lock loop in electronics. which is exactly the completely-integrable equation of the pendulum [2]. It has two kinds of solutions : oscillations in which the compass is locked with and oscillates around the rotating field with its own frequency $\omega_{0}(=\sqrt{F}$ when the magnitude of these oscillations is small) and rotations in which the compass is rotating at a rate different from that of the field. In phase space $(\theta, \dot{\theta})$, the lock-in or the resonance is associated with a set of closed trajectories (ellipsoidal for small oscillations), the largest closed trajectory being the separatrix. Similar results will be obtained with a second rotating field whose resonance will be symmetric with the first one about the axis $\theta=0$. If we now consider the two fields simultaneously, we easily understand that two fundamental resonances will appear in phase space, symmetric about the axis $\theta=0$. However, this is only true as long as the perturbation level is small, the system is no longer integrable, the phase space becomes three dimensional and stochastic trajectories appear. It is possible to return to the former phase space $(\theta, \theta)$, through a Poincare transformation. The K.A.M. theorem [3] means that the two resonances that we have already described remain stable as long as the perturbation level is not too high. The perturbation level is defined by the stochasticity parameter $s=(2 \sqrt{F} / \omega)$ which measures the width, in phase space, of the resonances compared with their separation. When $s=1$, the separatrix of the two unperturbed resonances touch each other, the resonances overlap, this is the criterion 
proposed by Chirikov [2], for the occurrence of large scale stochasticity.

The trajectories in the phases space at $s=0.68$ are given in figure 1 ; we find a number of resonances of which the larger $A$ and $A^{\prime}$ are just those we have described. The resonances $B$ and $B^{\prime}$. correspond to oscillations of the compass with $\langle\dot{\theta}\rangle=0$. On the other hand, the phase space also contains diffuse areas, growing like $s$ at the expense of the resonances, which also combine so allowing the system to diffuse into a larger and larger areas of phase space. When the system passes from resonance $A$ to resonance $A^{\prime}$, large scale stochasticity occurs.

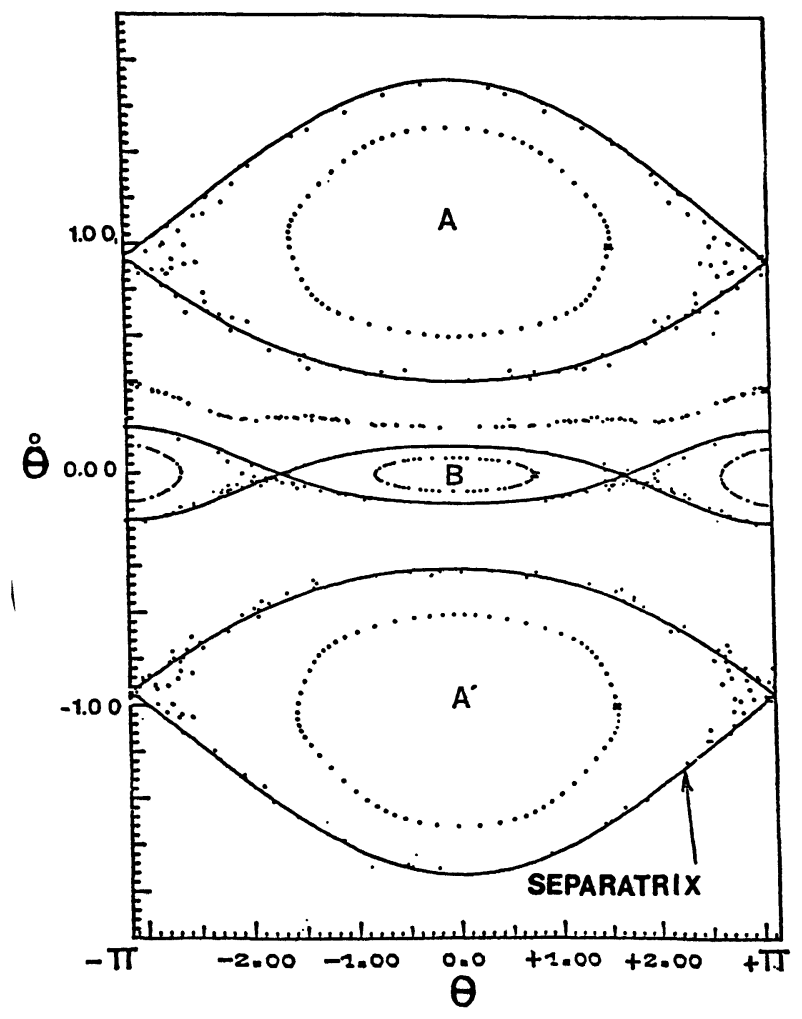

Fig. 1. - Calculated shape of the phases space at $s=0.68$. (We thank D. Escande and F. Doveil for this document.)

1. Experimental lay-out. - A compass, fixed on an axis guided by two bearings, is placed between two coils in a Helmholtz configuration fed by an alternating current. The stochasticity parameter $s$ is fixed by adjusting the magnitude of this current. The data acquisition system consists of a pick-up coil placed near the compass perpendicular to the excitation coils. The signal so induced is then Fourier analysed.

An important feature of our device is that it is a dissipative system. However, the friction is small, and the contraction, in phase space, slow so that we will be able to draw conclusions on the phase space of the associated Hamiltonian system, provided that the « trajectories » of the compass are considered over a short time interval. It is possible to model $\left({ }^{2}\right)$ our device with the equation :

$$
\ddot{\theta}+\alpha \dot{\theta}=-F[\sin (\theta-\omega t)+\sin (\theta+\omega t)]
$$

with $(\alpha / \omega) \simeq 3 \times 10^{-2}$.

2. Study of the destabilization of resonances. - In this study, the dissipative property plays a fundamental role : it allows an automatic centring on the elliptical point associated with each resonance to be achieved, so that it will be possible to follow the bifurcation cascade associated with the destabilization of these resonances.

Let us consider, to begin with the fundamental resonances $\mathrm{A}$ and $\mathrm{A}^{\prime}$. They correspond to a synchronous rotation with one of the rotating fields. The second rotating field acts as a perturbation at the frequency $(2 \omega / 2 \pi)$. Experimentally we observed that if $s$ is smaller than $1.74 \pm 0.02$, this perturbation does not destroy the elliptic point in the phase space. Nevertheless, a great number of lock-ins between the oscillations of the compass at $\omega_{0}$ and the perturbation at $2 \omega$ can occur. They appear when $\omega_{0}=(m .2 \omega / n)$. In this case the system has to be acted on in order that they may set in. When $s$ is increased these lock-ins appear with a well-defined hierarchy corresponding to increasing $\omega_{0}$; for instance, when $s \geqslant 0.88$, or $s \geqslant 1.05$ or $s \geqslant 1.36$ we find that $\omega_{0}$ is respectively $2 \omega / 5,2 \omega / 4,2 \omega / 3 \mathrm{t}$. In the case of the lock-in with $(2 \omega / 2)$ which appears when $s \geqslant 1.74$, the elliptic point splits and the compass, which until then was oscillating at the frequency $(2 \omega / 2 \pi)$ from side to side of the rotating field, now oscillates with the frequency $(\omega / 2 \pi)$. The experimental evidence for this phenomenon is slightly complicated by the rotational motion of the compass : this rotation induces a signal whose frequency itself is $(\omega / 2 \pi)$. We have, in fact, to monitor the magnitude of the second harmonic whose sudden growth reveals the beating of the rotation at $\omega$ and the oscillation also at $\omega$. From this bifurcation, the same scenario repeats itself on a smaller scale and possible lock-ins will now affect the oscillation motion at $\omega$. The two elliptic points are stable until $s=1.93$, when a new bifurcation with period doubling occurs. It simply corresponds to the occurrence of the subharmonic $(2 \omega / 4)$ (Fig. $2 a)$ on the same scenario, but this time the scale of the motion becomes very small and the corresponding parameter variations become extremely restricted. Experimentally it is difficult to increase this parameter without getting a lock-in $1 / 3(2 \omega / 12)$, which rapidly leads the motion of the compass to large scale chaos (for $s \simeq 1.94$ ). One fascinating property of this device is that it seems that the destabilization of all the resonances follow the same scenario.

$\left({ }^{2}\right)$ In fact, dissipation results from solid friction, so this model is somewhat crude. 

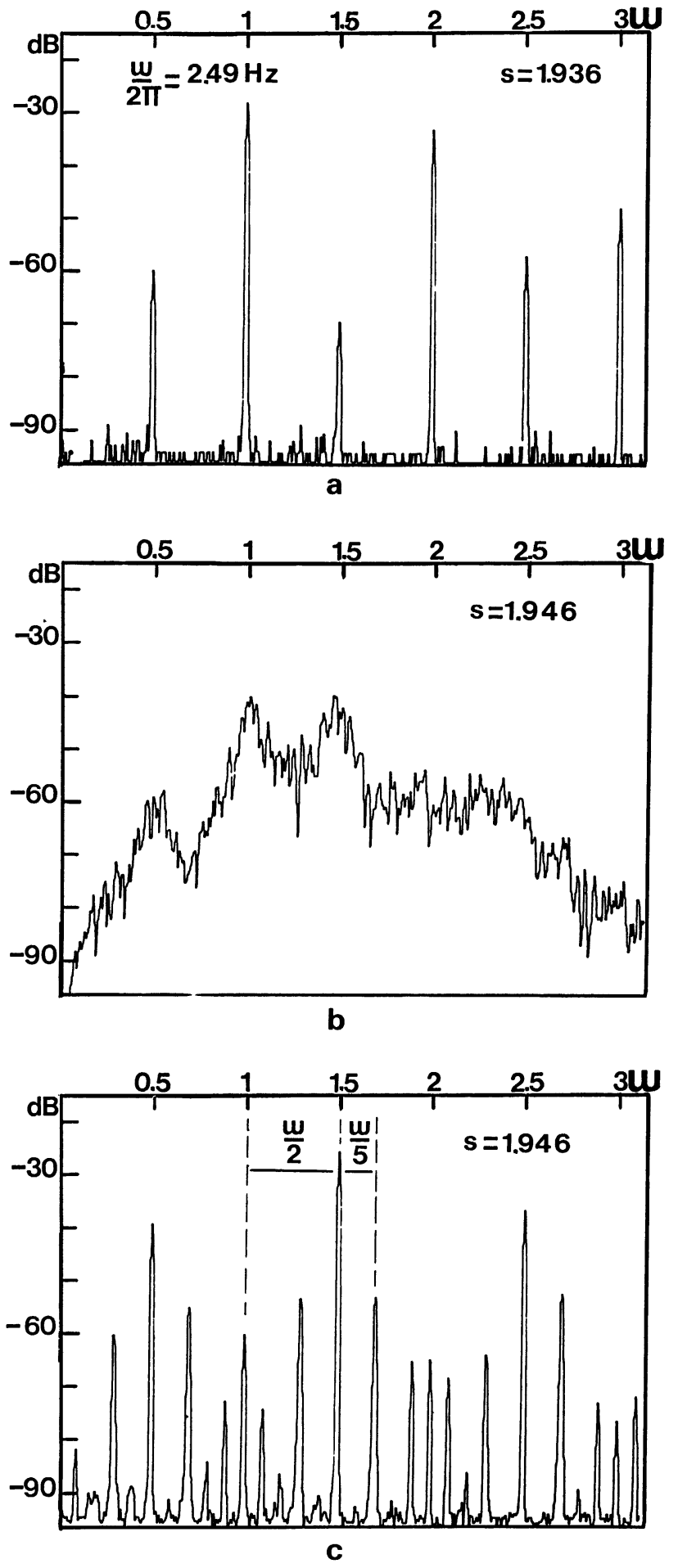

Fig. 2. - Pick-up coil signal spectra. a) After two period doublings ; b) Chaotic phase; c) After a new lock-in.
For example with resonance B, $\omega / 2$ appears for $s=0.92, \omega / 4$ for $s=1.04$.

3. Study of chaotic motion. - The occurrence of chaos from a lock-in appears in a first phase during which the system is nearly locked but exhibits abnormal amplitudes. It slowly escapes, from the lock-in, after about ten periods. This phase is followed by a phase of large scale chaos where the motion of the compass appears totally erratic. The compass rotates a few times along with one of the fields, then suddenly reverses its rotation, stops, starts again and so on. We think that such a motion illustrates the large scale stochasticity of Hamiltonian systems since the rotation inversions indicate that the chaotic motions are of large extension in the phase space. Further, these inversions may occur after a few periods of excitation only, which is a very short time compared with the time related to the dissipation (typically 100 periods). Beside this, the nature of this chaos seems to be Hamiltonian as shown by the spectra of figure $2 b$ where it can be seen that the noise merges at once in all the spectra, which is contrary to the usual experimental observations in dissipative systems [4]. Nevertheless the dissipation plays a fundamental role : this kind of chaos does not seem to be stable and the system finally finds a new lock-in which may be very complex as figure $2 c$ shows. This illustrates the attractive property of the elliptic points in dissipative systems. However such chaotic phases exhibit a great variety of durations, under the same conditions, from five minutes to a few hours ! We also notice that new resonances appear at a finite $s$ value. In the case of figure $2 c: s \simeq 1.92$.

We think that, even though the large scale stochasticity is a property of Hamiltonian systems, it also occurs in a transient way in slightly dissipative systems. The experimental device that we describe allows quantitative comparison with new theories in both dissipative and Hamiltonian systems. Thus we hope, that in a near future, we will be able to extract an experimental value for the $\delta$ exponent of Feigenbaum [5] in both cases.

Acknowledgments. - We are grateful to D. Escande, F. Doveil, S. Aubry, Y. Pomeau, P. Bergé and M. Dubois for stimulating discussions. We also thank M. Labouise and B. Ozenda for their technical assistance.

\section{References}

[1] Escande, D. F. and Doveil, F., Phys. Lett. 83A (1981) 307.

[2] Chirikov, B. V., Phys. Rep. 52 (1979) 263-379.

[3] Arnold, V. I. and Avez, A., Ergodic problems of classical mechanics (Benjamin, New York) 1968.

[4] Dubois, M. and Bergé, P., J. Physique 42 (1981) 167. Gollub, J. P. and Benson, S. V., J. Fluid. Mech. 100 (1980) 449
LibChaber, A. and MaUrer, J., NATO-ASI Proceedings, 1981, to be published.

[5] Feigendaum, M. J., Phys. Lett. 76 (1979) 375.

[6] Mclaughlin, J., J. Stat. Phys. 24 (1981) 377.

[7] Huberman, B. A., Crutchfield, J. P. and Packard, N. H., Appl. Phys. Lett. 37 (8) (1980) 750. 